\title{
Pharmakotherapie bei Neonaten und Jungtieren
}

\author{
Meike Mevissen \\ Abteilung Veterinär-Pharmakologie und Toxikologie, Departement Clinical Research and Veterinary Public Health, \\ Vetsuisse-Fakultät Universität Bern, Bern, Schweiz
}

Eine rationale Therapie beim Neugeborenen verlangt nicht nur Kenntnisse über die Pharmakologie (Wirkungsmechanismus, unerwünschte Wirkungen usw.) einzelner Arzneimittel, sondern ebenfalls Kenntnisse über Interaktionen zwischen mehreren Arzneimitteln und deren Pharmakokinetik unter Berücksichtigung der vorliegenden Erkrankung sowie der sich verändernden physiologischen Parameter beim Jungtier.

Die Daten zur Pharmakokinetik, die für einen in der Therapie eingesetzten Stoff erhoben werden, entstammen zumeist Studien an erwachsenen und gesunden Tieren. Diese Daten sind nicht in vollem Umfang auf die Verhältnisse von Jungtieren übertragbar.

Generell gilt, dass Arzneistoffe Biomembranen meist durch passive Diffusion passieren. Die altersabhängige Entwicklung des Organismus beeinflusst die Resorption, da der pH-Wert im Magen beim Neonaten in der Regel höher ist als beim Adulten. Aus diesem Grund können teilweise auch säurelabile Substanzen (z.B. Benzylpenicillin), die bei der Magenpassage des adulten Tieres zerstört werden, im Dünndarm des Neugeborenen resorbiert werden. Weiterhin ist die Entleerungszeit des Magens beim Jungtier länger, die Darmperistaltik funktioniert noch nicht im vollen Umfang und die intestinale Durchblutung nimmt altersabhängig zu. Die niedrige postnatale Expression von Effluxtransportern im Gastrointestinaltrakt und an der Blut-Hirn-Schranke bedingt beim Jungtier eine erhöhte orale Resorption sowie eine erhöhte Aufnahme in das Zentralnervensystem während der ersten Lebenswochen. Von besonderer Bedeutung ist auch die Entwicklung der Mikroflora im Darm, die insbesondere durch den Einsatz von Antibiotika nach oraler Gabe erheblich gestört wird. Qualitative Unterschiede in der Diät des Neugeborenen können ebenfalls dramatische Effekte auf die Resorption im Gastrointestinaltrakt haben. Die gleichzeitige Applikation von Antibiotika mit Milchaustauschern kann zu einer geringeren Bioverfügbarkeit und somit $\mathrm{zu}$ Wirkungsverlusten führen. Die orale Gabe von Tetracyclinen zusammen mit Milchaustauschern bedingt eine Chelatbildung mit Calcium in der Milch.

\section{KARGER}

Fax +497614520714

Information@Karger.com

www.karger.com
Fazit: Eine genaue Vorhersage des zu erwartenden Umfanges der Resorption eines Arzneimittels ist daher kaum möglich, da sowohl eine gesteigerte als auch eine verminderte Resorption auftreten kann. Bei Kenntnis der Einflussgrössen und Faktoren lässt sich somit nur eine Abschätzung hinsichtlich gesteigerter oder minimierter Resorption durchführen.

Da die Wirkung eines Arzneimittels von der Konzentration am Ort der Wirkung abhängt, bestimmt die Verteilung des Stoffes im Organismus wesentlich die Wirksamkeit und den Therapieerfolg. Die Proteinbindung unterliegt altersabhängigen Veränderungen, aber auch pathophysiologische Prozesse können die Proteinbindung beeinträchtigen. Insbesondere ist somit bei Verwendung von Substanzen mit hoher Proteinbindung (z.B. nichtsteroidale antiinflammatorische Arzneimittel (NSAID)) eine Verstärkung der Wirkung beim Jungtier zu beobachten.

Einer Altersabhängigkeit unterliegt ebenfalls die Zusammensetzung des Organismus. Der Wassergehalt des Körpers (ca. 75\% Neugeborene, ca. 50-60\% Adulte), insbesondere jener des Extrazellularraumes, nimmt altersabhängig ab (2:1 extrazellulär/intrazellulär beim Neugeborenen; 1:2 beim Adulten). Somit sind hydrophile Substanzen, wie z.B. Benzylpenicillin sowie Cephalosporin- und AminoglykosidAntibiotika, schlechter wirksam, da eine grössere Verteilung im Organismus des Jungtieres vorliegt. Während der Körperwassergehalt abnimmt, nimmt der Fettanteil des Organismus zu. Lipophile Stoffe sind beim Jungtier aufgrund der geringeren Einlagerung in das Fettgewebe stärker wirksam.

Eine vermehrte Durchlässigkeit der Blut-Hirn-Schranke beim Neugeborenen bedingt, dass zentralwirksame Pharmaka stärkere Wirkungen und - besonders zu beachten - auch stärkere unerwünschte Wirkungen haben.

Eine Folge der beim Jungtier eingeschränkten Metabolisierung kann also eine verlangsamte Abnahme der Konzentration im Organismus sein. Die postnatale Reifung der Substanz-metabolisierenden Enzyme in der Leber verläuft biphasisch, bestehend aus einem schnellen und linearen Anstieg der Aktivität der Enzyme während der ersten 3-4 
Tab. 1. Konsequenzen der altersabhängigen Veränderungen für den Einsatz der Arzneimitteltherapie beim Jungtier

Insbesondere in den ersten 3-4 Lebenswochen ist bei der Arzneimitteltherapie zu beachten:

a) Vorsicht bei Arzneimitteln mit geringgradiger therapeutischer Breite, insbesondere wenn sie überwiegend renal eliminiert werden.

b) Bei gut wasserlöslichen (polaren) Arzneimitteln ist aufgrund des relativ grösseren Extrazellularraumes oft eine höhere Dosis erforderlich.

c) Aufgrund geringgradiger metabolischer Aktivität und eingeschränkter Nierenfunktion sind oft grössere Dosisintervalle notwendig.

d) Einige Arzneimittel sollten mit Vorsicht angewendet werden, da besondere pharmakokinetische oder pharmakodynamische Eigenschaften des Arzneimittels zu beachten sind: Einlagerung in Knochen und Zähne, z.B. Tetracycline; Knorpelschäden (Chondropathie) durch Gyraseinhibitoren (z.B. Enrofloxacin).

Lebenswochen sowie einer nachfolgenden verlangsamten Steigerung bis zur 10. Woche post partum. Somit können toxische Konzentrationen bereits bei vorschriftsmässiger Verabreichung von Arzneimitteln (wie vorgeschrieben) auftreten. Gleichzeitig kann aber auch eine geringgradige Metabolisierung die Wirkung von Arzneimitteln vermindern, die als sogenannte «Prodrugs» verabreicht werden $[1,2]$.

Die renale Elimination stellt den wichtigsten Eliminationsweg der meisten Arzneimittel dar. Die Ausscheidung der Muttersubstanz sowie deren jeweilige Metaboliten werden meist durch glomeruläre Filtration ausgeschieden. Prozesse der renalen Elimination, wie die glomeruläre Filtration und insbesondere die tubuläre Sekretion, erreichen bei fast allen
Haustieren erst mehrere Wochen bis Monate post partum die volle Leistungsfähigkeit. Die Durchblutung des renalen Gewebes nimmt ebenfalls altersabhängig zu, was die Eliminationsleistung beeinflusst. Somit ergeben sich verlängerte Halbwertszeiten bei Jungtieren für Substanzen, die via Metabolisierung wasserlöslicher gemacht und renal eliminiert werden.

Neben pharmakokinetischen Unterschieden zwischen Jungtieren und adulten Tieren sind auch pharmakodynamische Unterschiede beschrieben, die insbesondere zentrale sowie vegetative Funktionen betreffen (Tab. 1). Die Regulation der Körpertemperatur erfolgt noch unvollständig. Kolostrum ist kritisch für die metabolischen Anforderungen der Wärmeregulation im neonatalen Organismus. Die Aufnahme von Kolostrum in den ersten Lebensstunden ist von entscheidender Bedeutung bei Neugeborenen. Die Fähigkeit zur Resorption von kolostralen Antikörpern nimmt rasch ab [3].

Die konventionelle Therapie birgt Vor- und Nachteile, z.B. bei der Septikämie des neugeborenen Fohlens, für die Mortalitätsraten von bis zu $68 \%$ beschrieben sind [4-6]; diesbezüglich stellt auch der Einsatz von Arzneipflanzen eine Möglichkeit dar [7-9]. Ein weiterer Aspekt besteht in der Aktivierung antimikrobieller Peptide bei Jungtieren [10-15].

\section{Disclosure Statement}

Für die Autorin bestehen keine Interessenkonflikte.

\section{Literatur}

1 Gow PJ, Ghabrial H, Smallwood RA, Morgan DJ, Ching MS: Neonatal hepatic drug elimination. Pharmacol Toxicol 2001;88:3-15.

2 Schwark WS: Factors that affect drug disposition in food-producing animals during maturation. J Anim Sci 1992;70:3635-3645.

3 Löscher WK, Kietzmann M: Pharmakotherapie beim Welpen - einfach nur eine geringere Dosis? Kleintier konkret 2012;15:8-13.

4 Brumbaugh GW: Neonatal adjustments. Vet Clin North Am Food Anim Pract 2003;19:551556.

5 Hoskins JD: Feline neonatal sepsis. Vet Clin North Am Small Anim Pract 1993;23:91-100.

6 Moon PF, Massat BJ, Pascoe PJ: Neonatal critical care. Vet Clin North Am Small Anim Pract 2001;31:343-365.

7 Vendrig JC, Fink-Gremmels J: Intestinal barrier function in neonatal foals: options for improvement. Vet J 2012;193:32-37.
8 Passari AK, Mishra VK, Saikia R, Gupta VK, Singh BP: Isolation, abundance and phylogenetic affiliation of endophytic actinomycetes associated with medicinal plants and screening for their in vitro antimicrobial biosynthetic potential. Front Microbiol 2015;6:273.

9 Trend S, Strunk T, Hibbert J, et al: Antimicrobial protein and peptide concentrations and activity in human breast milk consumed by preterm infants at risk of late-onset neonatal sepsis. PloS One 2015;10:e0117038.

10 de Souza Candido E, e Silva Cardoso MH, Sousa DA, et al: The use of versatile plant antimicrobial peptides in agribusiness and human health. Peptides 2014;55:65-78.

11 Brown D: Antibiotic resistance breakers: can repurposed drugs fill the antibiotic discovery void? Nat Rev Drug Discov 2015;14:821-832.
12 Holaskova E, Galuszka P, Frebort I, Oz MT: Antimicrobial peptide production and plantbased expression systems for medical and agricultural biotechnology. Biotechnol Adv 2015; 33:1005-1023.

13 Mansour SC, Pena OM, Hancock RE: Host defense peptides: front-line immunomodulators. Trends Immunol 2014;35:443-450.

14 Rocha-Ferreira E, Hristova M: Antimicrobial peptides and complement in neonatal hypoxiaischemia induced brain damage. Front Immunol 2015;6:56.

15 Takahashi D, Shukla SK, Prakash O, Zhang G: Structural determinants of host defense peptides for antimicrobial activity and target cell selectivity. Biochimie 2010;92:1236-1241. 